OPEN ACCESS

Edited by:

Yufei Ma,

Harbin Institute of Technology, China

Reviewed by:

Pavel Honzatko,

Institute of Radio Engineering and Electronics (ASCR), Czechia

Bo Guo,

Harbin Engineering University, China

*Correspondence:

Baole Lu

lubaole1123@163.com

Jintao Bal

baijt@nwu.edu.cn

Specialty section:

This article was submitted to

Optics and Photonics,

a section of the journal

Frontiers in Physics

Received: 04 November 2021 Accepted: 30 November 2021

Published: 21 December 2021

Citation:

Zuo Z, Wang K, Chen H, Lu B and BaiJ (2021) Recent Progress and Development Trend of Self-Sweeping

Fiber Laser.

Front. Phys. 9:809049.

doi: $10.3389 /$ fphy.2021.809049

\section{Recent Progress and Development Trend of Self-Sweeping Fiber Laser}

\author{
Zhenzhong Zuo ${ }^{1,2}$, Kaile Wang ${ }^{1,2}$, Haowei Chen ${ }^{1,2,3}$, Baole $L u^{1,2,3 *}$ and Jintao Bai ${ }^{1,2,3 *}$ \\ ${ }^{1}$ State Key Laboratory of Energy Photon-Technology in Western China, Shaanxi Engineering Technology Research Center for \\ Solid State Lasers and Application, Northwest University, Xi'an, China, ${ }^{2}$ Institute of Photonics and Photon-Technology, \\ Northwest University, Xi'an, China, ${ }^{3}$ International Collaborative Center on Photoelectric Technology and Nano Functional \\ Materials, Provincial Key Laboratory of Photo-Electronic Technology, Northwest University, Xi'an, China
}

Since 2011, when Kir'yanov et al. first reported a new wavelength self-sweeping ytterbiumdoped fiber laser that does not rely on any tuning element but only on the dynamic induced grating generated in the gain fiber by the standing wave resonator structure, the selfsweeping effect based on fiber waveguides has been extensively studied, leading to great progress in fundamental physics and other applications of self-sweeping fiber lasers. Different doped fiber lasers have not only achieved the self-sweeping effect, but also observed new phenomena such as anomalous self-sweeping and continuous pulses. Due to their remarkable spectral and pulsed characteristics, self-sweeping fiber lasers have been widely used in spectral detection, fiber sensing and short pulse synthesis. In this paper, we will introduce the classification of different doped self-sweeping fiber lasers, summarize their different implementations, and introduce their self-sweeping laws, pulse characteristics, recent progress of applications and future development prospects.

Keywords: fiber laser, self-sweeping effect, wavelength tunable, spectral characteristics, pulse dynamic

\section{INTRODUCTION}

Tunable fiber lasers are valuable for optical communication, optical sensing, and spectral synthesis due to their flexible wavelength tuning properties [1-5]. Among them, swept-frequency lasers with periodic wavelength variation using an optical filter with an electric driver are widely used in fiber optic sensing, biomedicine, and spectral detection. However, the development of swept-frequency fiber lasers is limited by the influence of swept-frequency devices [6-8] and the output performance of the lasers themselves, and in recent years, a new swept-frequency fiber laser based on the selfsweeping effect has attracted a lot of attention from researchers. The self-sweeping effect refers to the spontaneous, stable and periodic tuning process of the laser output wavelength in a certain spectral range, and does not require any complex tunable devices or electric drives. It is attributed to the spatial hole-burning (SHB) effect of the gain fiber in the standing wave field [9], which forms a periodic light intensity distribution, and then the particle number inversion causes a refractive index change, which induces a grating-like structure along the gain fiber, i.e., a "gain grating and a phase grating" [10-12], where the dynamic change in the net gain of the grating resulted in a shift of the spectrum. The self-sweeping effect was first reported in 1962 [13], where a periodic shift in wavelength was observed in a ruby laser. Half a century later, based on the excellent waveguide medium of optical fiber, Ref.[14] in Russia successfully observed the self-sweeping effect in an ytterbium-doped fiber laser and named it as self-sweeping fiber laser.

Since the formal report on self-sweeping ytterbium-doped fiber lasers in 2011, they have undergone rapid development in the past decade. So far, based on the typical Fabry-Perot (F-P) linear resonator structure, the self-sweeping effect has been observed in different doped fiber lasers 


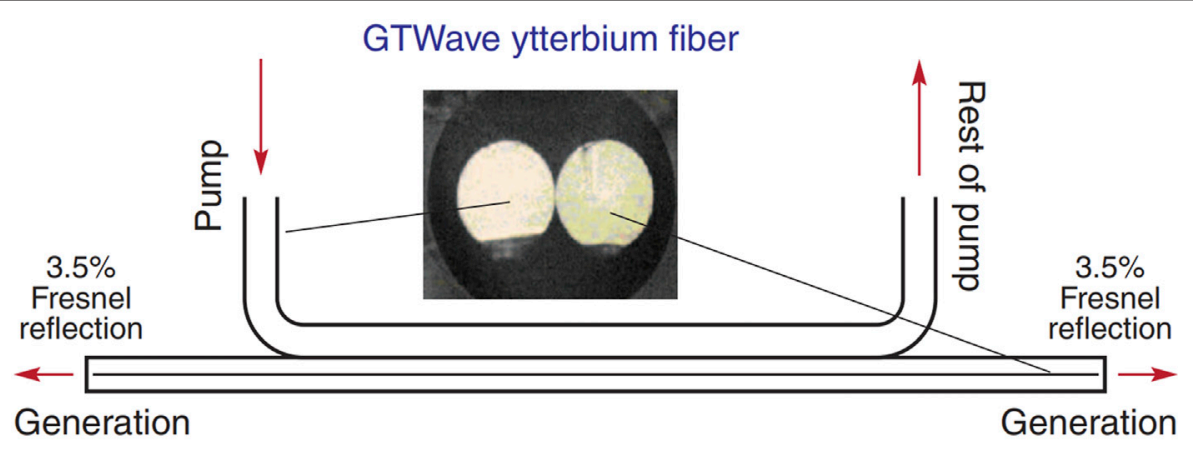

FIGURE 1 | Experimental setup of Yb-doped fiber laser generating self-sweeping effect for the first time [14]. (Inset) A cross-sectional view of a GT-Wave fiber.

such as ytterbium, erbium, thulium, thulium-holmium, bismuth, holmium and neodymium. In addition, self-sweeping ranges up to $45 \mathrm{~nm}$ have been obtained in ytterbium-doped fiber lasers by temperature-controlled Lyot filters; single-frequency selfsweeping fiber lasers have been achieved by limiting the intracavity longitudinal mode number by adjusting the resonator length; and normal as well as reverse self-sweeping effects have been defined according to the wavelength shift direction in hybrid self-sweeping fiber lasers. Later, both bidirectional and unidirectional fiber ring resonator structures were used to realize self-sweeping fiber lasers, while the timedomain dynamics evolved from the pulsed state to the continuous wave state. The rapid development of self-sweeping fiber lasers has promoted their applications in various research fields such as spectral detection and analysis, pulsed coherent synthesis, and optical sensing, and has become one of the research hotspots for fiber lasers.

Combined with the research work of our team in self-sweeping fiber laser, this paper summarizes the research status and latest application progress of self-sweeping fiber laser at home and abroad from four aspects: doped fiber types, pulse intensity dynamics, basic law of self-sweeping and application of selfsweeping fiber laser, and looks forward to its future development direction.

\section{DIVERSELY DOPED SELF-SWEEPING FIBER LASERS \\ Ytterbium-Doped Self-Sweeping Fiber Laser \\ Linear Resonator}

In 2011, the Russian Kir'yanov' research group [14] obtained the first ytterbium-doped self-sweeping fiber laser using the F-P linear resonator structure. The principle of this experimental setup is shown in Figure 1. The gain medium of the laser is a special section of GT-Wave ytterbium-doped fiber with the presence of two core channels for the transmission of pump light and signal light, respectively. The pump light is pumped into the ytterbium-doped fiber to produce an excited amplified spontaneous emission (ASE) spectrum into the other channel of the fiber. The resonant cavity of the laser consists of two flat tangent ports of the fiber providing 3.5\% Fresnel reflection on the left and right side, and the interference of the standing wave field inside the resonator forms a SHB effect producing a dynamic grating-induced self-sweeping effect.

The tuning of the output spectrum in the range of 1,081 $\mathrm{nm}-1087 \mathrm{~nm}$ was observed at pump powers of $1.4,1.7,2.0,2.3$, 2.6, and $2.9 \mathrm{~W}$, respectively. When the pump power is increased from 1.4 to $2.6 \mathrm{~W}$, the self-sweeping spectral range gradually increases to $7 \mathrm{~nm}$, but further increasing the pump power, the stable self-sweeping spectrum is replaced by a random chaotic spectrum due to the presence of a very pronounced stimulated Raman scattering (SRS) signal at higher pump power that destroys the conditions for the stable generation of the selfsweeping effect.

Following the report by Kir'yanov's group, the self-sweeping effect in a typical F-P linear resonator structure was also achieved in ytterbium-doped fiber laser by Lobach's group [15] at Novosibirsk University, Russia, in 2011, with the experimental setup shown in Figure 2A, where the high reflectance mirror is formed by the fiber loop mirror (FLM), while the output mirror is formed by the Fresnel reflection from a perpendicularly cleaved fiber facet. The polarization controller (PC) is adjusted for a selfsweeping range up to $16 \mathrm{~nm}(1,062 \mathrm{~nm}-1078 \mathrm{~nm})$ and a selfsweeping rate range of $0.5 \mathrm{~nm} / \mathrm{s}-16 \mathrm{~nm} / \mathrm{s}$, as shown in Figure $2 B$. After replacing the FLM with a fiber bragg grating (FBG) with a central wavelength of $1,066 \mathrm{~nm}$, the maximum self-sweeping range is reduced to $0.1 \mathrm{~nm}$ due to the bandwidth limitation of the FBG. However, due to the significant emission loss of the laser in Figure 2A, this greatly affects the stability of the self-sweeping phenomenon. To solve this problem, in 2012 they [16] used an all-fiber structure and obtained a stable self-sweeping range of about $8-10 \mathrm{~nm}$, where the self-sweeping range was up to $16 \mathrm{~nm}$ under specific PC modulation. In addition, the linewidth of the self-sweeping fiber laser was very narrow, about $0.3 \mathrm{pm}$, which was much smaller than the $0.1 \mathrm{~nm}$ linewidth obtained by Ref.[14]. In 2013, Ref. [17] extended the self-sweeping band to 1,087 $\mathrm{nm}-1094 \mathrm{~nm}$ with a slow self-sweeping rate of $0.24 \mathrm{~nm} / \mathrm{s}$.

Based on the experiments of Lobach et al. in 2013, Peterka's team [18] from the Czech Academy of Sciences obtained the selfsweeping effect near the $1,080 \mathrm{~nm}$ band by adjusting the current and shell temperature of the intracavity laser diode (LD) in the selfsweeping range of $2 \mathrm{~nm} / \mathrm{s}-6 \mathrm{~nm} / \mathrm{s}$, which can reach $7 \mathrm{~nm}$ when the 

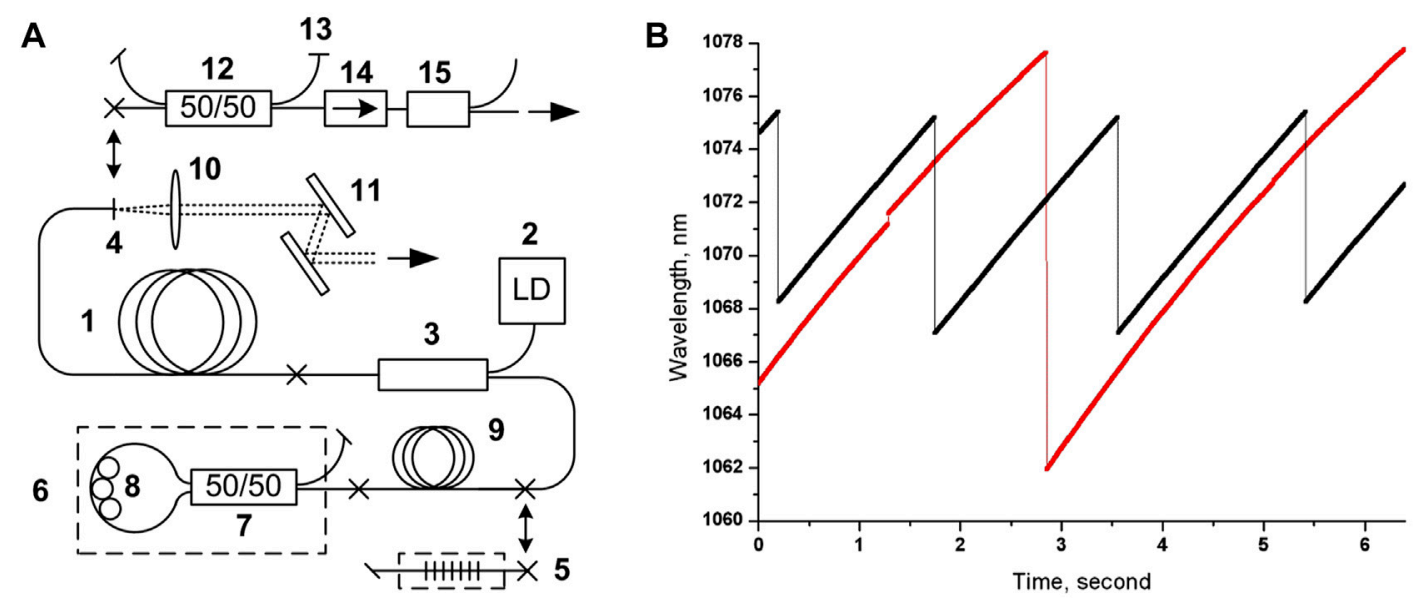

FIGURE 2 | (A) The experimental setup of a typical F-P cavity Yb-doped self-sweeping fiber laser [15] (1 Yb-doped fiber; 2 Laser diode; 3 Pump combiner; 4 Cleaved; 5 Fiber bragg grating; 6 Fiber loop mirror; 7 50/50 coupler; 8 Polarization controller; 9 Single-mode fiber; 10 Aspherical lens; 11 Dichromatic mirror; 12 50/50 coupler; 13 Cleaved; 14 Isolator; 15 Coupler); (B) FLM cavity spectral dynamic (Red-black curve corresponding to different modulations of PC).

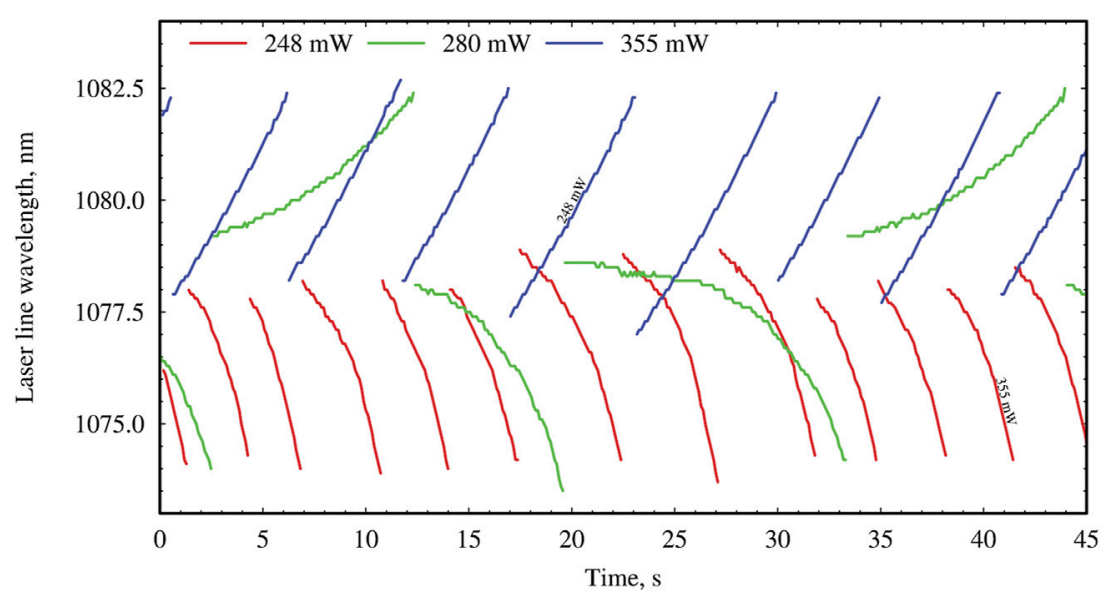

FIGURE 3 | Three spectral dynamics observed by Yb-doped self-sweeping fiber laser in 2017 [22]. Normal self-sweeping (blue curve); Mixed self-sweeping (green curve); Reverse self-sweeping (red curve).

LD shell temperature is $35^{\circ} \mathrm{C}$. In 2016, the Lobach's team [19] obtained a self-sweeping in a wavelength range of up to $18 \mathrm{~nm}$ with a central wavelength adjustable from $1,025 \mathrm{~nm}$ to $1,070 \mathrm{~nm}$ by varying the length of the gain fiber. The self-sweeping range can be tuned to below $30 \mathrm{~nm}$ by simultaneously varying the resonator loss and pump power. In addition, the maximum self-sweeping range can be up to $18 \mathrm{~nm}$ by adjusting the temperature of the gain fiber or the pump source, respectively. In the same year, the group [20] achieved a self-sweeping range of $1,058-1076 \mathrm{~nm}$ with a selfsweeping rate of $0.8 \mathrm{~nm} / \mathrm{s}$ and an output power greater than $50 \mathrm{~mW}$ for an ytterbium-doped self-sweeping fiber laser. In 2017, they [21] obtained a self-swept spectral tuning of $1,050-1070 \mathrm{~nm}$ at a pump power of $2 \mathrm{~W}$ with a self-sweeping rate of about $1 \mathrm{~nm} / \mathrm{s}$ and increased the average and peak output power to about 100 and $400 \mathrm{~mW}$.
In 2017, Ref. [22] achieved the first reverse self-sweeping effect of an ytterbium-doped fiber laser. In this experiment, the wavelength sweeping direction was divided into two categories, the normal self-sweeping direction, which refers to the shift of the output wavelength from short to long wavelengths, and the reverse self-sweeping direction, which refers to the shift of the output wavelength from long to short wavelengths. The ytterbium-doped fiber laser has three types of self-sweeping output spectral dynamics with pump power: normal selfsweeping (blue curve in Figure 3), reverse self-sweeping (red curve in Figure 3) and hybrid self-sweeping (unstable state where both normal and reverse self-sweeping exist, green curve in Figure 3). The normal self-sweeping range was $2.5-6.6 \mathrm{~nm}$ with a sweeping rate of $0.3 \mathrm{~nm} / \mathrm{s}-6.6 \mathrm{~nm} / \mathrm{s}$, and the reverse self-scan range was $4.3-5.3 \mathrm{~nm}$ with a rate of $0.5-1.7 \mathrm{~nm} / \mathrm{s}$. 

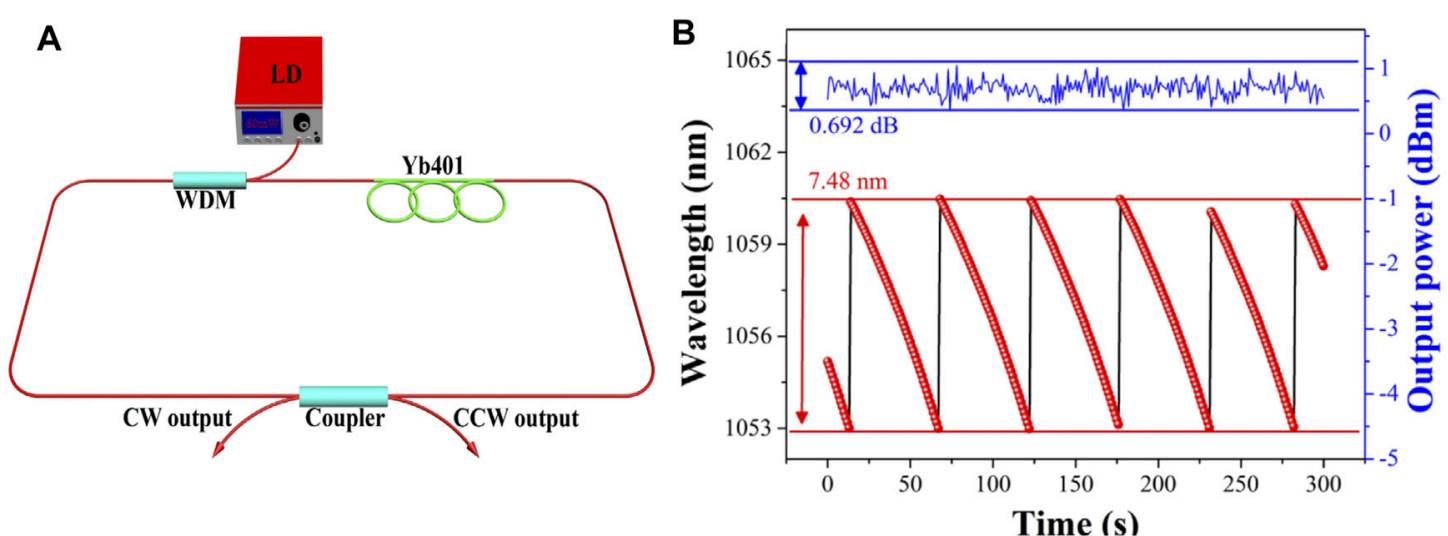

FIGURE 4 | (A) Experimental setup of all-polarization-maintaining bidirectional ring resonator Yb-doped self-sweeping fiber laser [25]; (B) Self-sweeping spectral dynamics and output power stability at pump power of $65 \mathrm{~mW}$.

\section{Bidirectional Fiber Ring Resonator}

In 2018, Sze Yun Set's group [23] at the University of Tokyo, Japan, achieved the first bidirectional fiber ring resonator selfsweeping fiber laser using thulium-doped fiber as the gain medium. In 2019, our group [24] achieved the reverse selfsweeping effect near the $1.037 \mu \mathrm{m}$ band in a bidirectional fiber ring resonator ytterbium-doped fiber laser with a single-mode structure. The laser achieved a self-scan range of $2.75 \mathrm{~nm}$ $(1,038.98-1036.23 \mathrm{~nm})$ with an average self-sweeping rate of $0.38 \mathrm{~nm} / \mathrm{s}$ for output spectral tuning at a pump power of $60 \mathrm{~mW}$. The output power in the stable self-sweeping state fluctuates by about $2 \mathrm{~dB}$ due to the influence of the external environment and the gain difference in the self-sweeping range.

Due to the narrow self-sweeping range generated by the singlemode structured bi-directional resonator ytterbium-doped selfsweeping fiber laser and the high output fluctuations due to the external environment, it is difficult to observe the full characteristics of the self-sweeping effect. To solve this problem, in 2020, our group [25] used an all-polarizationmaintaining resonator structure and two different coupling ratios (50/50 and 10/90) of the output coupler to achieve a comparative analysis of the self-sweeping effect, and the experimental setup is shown in Figure 4A. In the experiments, the self-scan range obtained with the output coupler of 50/50 coupling ratio was $7.48 \mathrm{~nm}$ at a pump power of $65 \mathrm{~mW}$ (red line in Figure 4B), the output power fluctuation was about $0.692 \mathrm{~dB}$ (blue line in Figure 4B), and the self-sweeping rate ranged from $7.8 \mathrm{~nm} / \mathrm{min}$ to $9.48 \mathrm{~nm} / \mathrm{min}$. When the output coupler of $10 / 90$ was used in the resonator, the self-sweeping rate and the pump power range could be effectively reduced to as low as $0.016 \mathrm{~nm} /$ $\mathrm{min}$. The reason for this reduction is that the microsecond pulses generated by the latter are often spaced at larger intervals.

Based on previous experiments, a bidirectional resonator ytterbium-doped self-sweeping fiber laser based on intracavity loss tuning brought by bending the fiber circle was investigated by our group [26] in 2021. The self-sweeping was obtained over intervals in the range $4-8 \mathrm{~nm}$ with a sweeping rate of

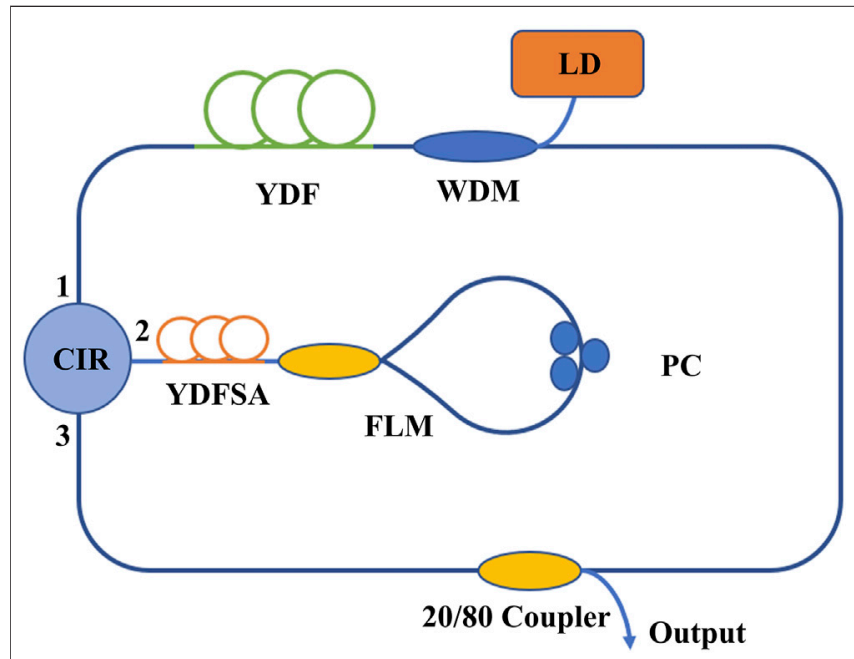

FIGURE 5 | Experimental setup of Yb-doped self-sweeping fiber laser with unidirectional ring cavity based on a fiber saturable absorber [28].

$0.19-0.43 \mathrm{~nm} / \mathrm{s}$ while the central wavelength was adjusted in a range of $1,055.6 \mathrm{~nm}-1034.6 \mathrm{~nm}$ by varying the bending loss. This experiment shows that the self-sweeping rate increases as the diameter of the bent fiber circle decreases, and the self-sweeping range tends to be further towards shorter wavelengths. This simple wavelength tuning method not only saves cost, but also helps to improve the sweeping performance of the self-sweeping fiber laser and expand its application in the field of spectral detection.

\section{Unidirectional Fiber Ring Resonator}

From the self-sweeping fiber lasers reported in recent years, it is known that the self-sweeping effect depends on the dynamic grating formed by the standing wave field in the resonator, whether in a linear resonator or a bidirectional fiber ring resonator structure, and based on this principle, we can try to 

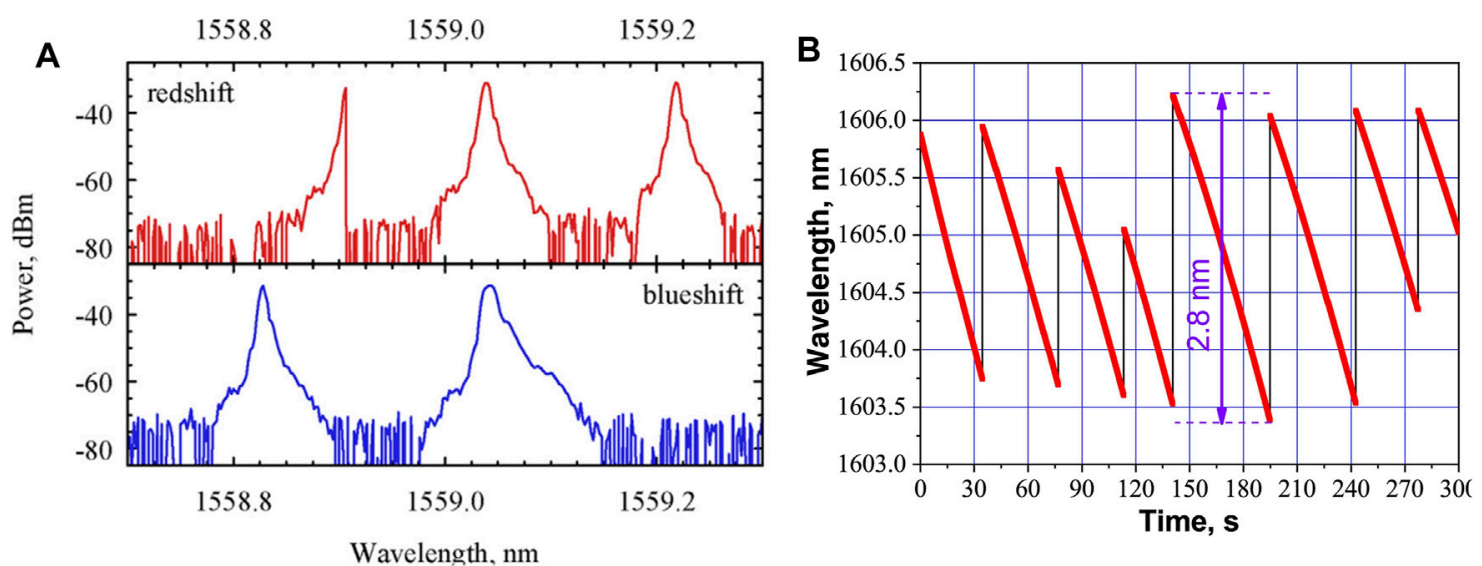

FIGURE 6 | Self-sweeping spectrum dynamics of Er-doped self-sweeping fiber laser. (A) Wavelength red-shift and blue-shift self-sweeping [29]; (B) Reverse selfsweeping [30].

form a dynamic grating in the fiber saturable absorber [27] to realize the self-sweeping effect.

In 2021, our group [28] introduced a $1.8 \mathrm{~m}$-long section of ytterbium-doped fiber as a fiber saturable absorber into the laser resonator through a circulator (CIR), and the experimental setup is shown in Figure 5. The ytterbium-doped fiber absorbs light in two directions to form a standing wave, forming a positiondependent periodic refractive index change, i.e., a grating, which in turn produces a normal self-sweeping effect. Also because of its filtering effect, as a tracking filter, the appearance of the laser's central output wavelength is also very dependent on it. The experiment obtained a self-sweeping spectral tuning of $1.5-4.85 \mathrm{~nm}$ with a self-sweeping rate of $0.145-0.284 \mathrm{~nm} / \mathrm{s}$. This work both extends the application of fiber saturable absorbers and provides a new direction for the research of self-sweeping fiber lasers.

\section{Erbium-Doped Self-Sweeping Fiber Laser}

Based on a typical F-P linear resonator structure, an erbiumdoped self-sweeping fiber laser was first reported by Peterka's group [29] in 2018, where mean wavelength was adjusted by a tunable filter in a range $1.54-1.57 \mu \mathrm{m}$. A $30 \mathrm{~cm}$ section of thulium-doped fiber was fused in the resonator as a passive Q-tuned switch, but the fiber did not achieve huge pulses like saturable absorbers, which exhibited a lower saturation intensity than expected by Peterka et al. Only the concomitant effect of selfsweeping-self-pulse effect-was achieved. Since the signal optical power in the thulium-doped fiber is very small and the inversion of the excited collective number of thulium ions is negligible, Peterka et al. suggest that the SHB effect may not occur in the thulium-doped fiber as in the erbium-doped fiber to form a dynamic grating to generate self-sweeping. The red and blue shifts of wavelengths were observed by the conditioning PC, as shown in Figure 6A. The self-sweeping ranges were 0.37 and $0.43 \mathrm{~nm}$, respectively, and the average self-sweeping rates were $2.85 \mathrm{~nm} / \mathrm{s}$ and $1.48 \mathrm{~nm} / \mathrm{s}$, respectively. In 2020, Lobach's group [30] implemented an erbium-doped self-sweeping fiber laser in the $1,605 \mathrm{~nm}$ band and extended the self-sweeping range to $2.8 \mathrm{~nm}$, as shown in Figure 6B.

\section{Thulium-Doped Self-Sweeping Fiber Laser Linear Resonator}

In 2018, the Lobach research group [31] achieved a stable selfsweeping phenomenon in the $1.92 \mu \mathrm{m}$ band for the first time using a thulium-doped fiber as a laser gain medium with a selfsweeping range of $21.5 \mathrm{~nm}$ and even $26 \mathrm{~nm}$ at some moments. They also reported [32] a hybrid thulium-doped self-sweeping fiber laser in 2019. This laser obtained three self-sweeping spectral dynamics in the presence of pump power: reverse self-sweeping (Figures 7A,B), wavelength stop state (Figure 7C, the wavelength can be stopped at any value in the 1912-1923 nm range) and normal self-sweeping (Figures 7D,E). The results show that any wavelength output in a specific spectral range of about $10 \mathrm{~nm}$ can be obtained by appropriately varying the pump power, which may contribute to the development and practical application of self-sweeping fiber lasers.

\section{Bidirectional Fiber Ring Resonator}

In 2018, a bidirectional fiber ring resonator thulium-doped selfsweeping fiber laser was first proposed by Sze Yun Set's team at the University of Tokyo, Japan [23], and its experimental principle is shown in Figure 8. In the absence of any direction-selective elements such as optical isolators in the laser resonator, the ASE spectrum generated by a pumped thulium-doped fiber amplified by an erbium-doped optical amplifier (EDFA) propagates CW and CCW along the resonator shape, creating a standing wave field in the thuliumdoped fiber, generating a $\mathrm{SHB}$ effect and generating a dynamic grating for self-sweeping. The laser achieved reverse selfsweeping near the $1970 \mathrm{~nm}$ band in the output spectral range from 10 to $15 \mathrm{~nm}$. In 2019, the research group [33] added PC to the resonator and obtained the bi-directional self-sweeping effect in the $1.95 \mu \mathrm{m}$ band by twisting the PC. This experiment showed that adjusting the PC could control the self-sweeping rate, and 


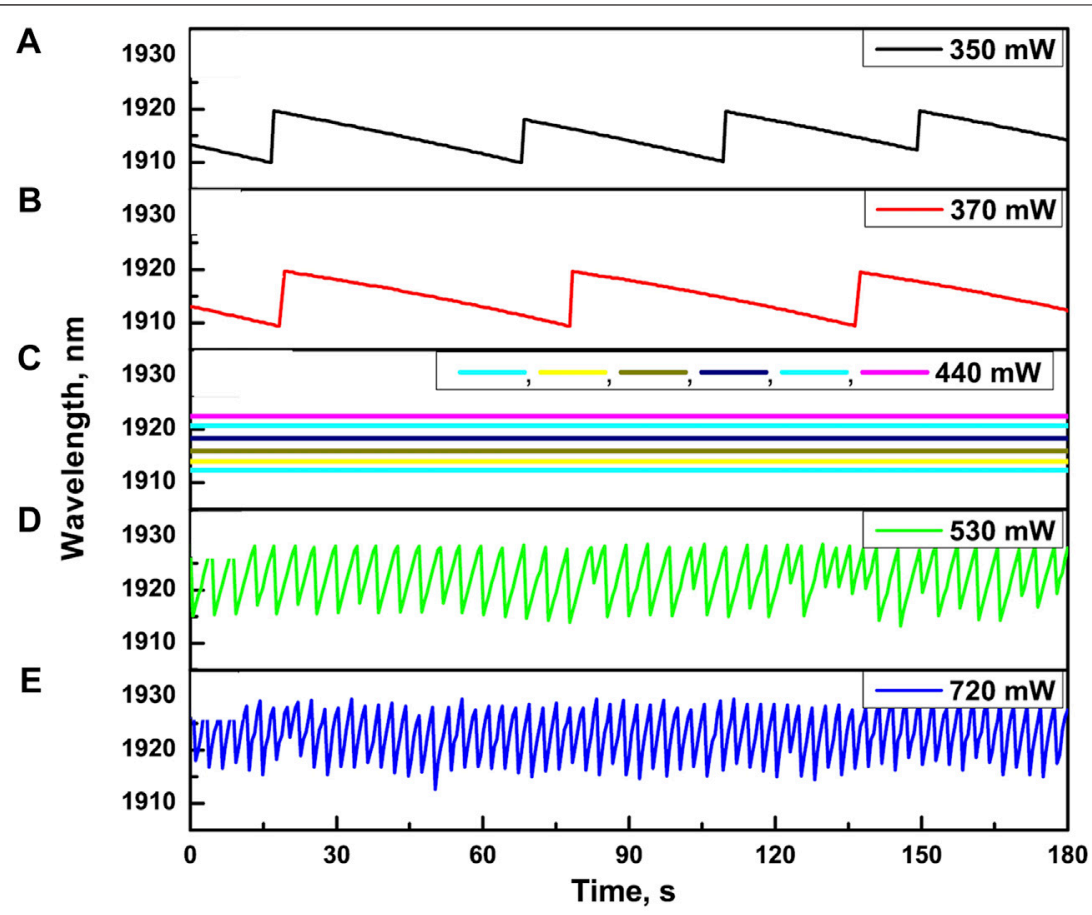

FIGURE 7 | Self-sweeping spectrum dynamics of hybrid Tm-doped self-sweeping fiber laser [32]. (A), (B) Reverse self-sweeping; (C) Wavelength stop state; (D), (E) Normal self-sweeping.

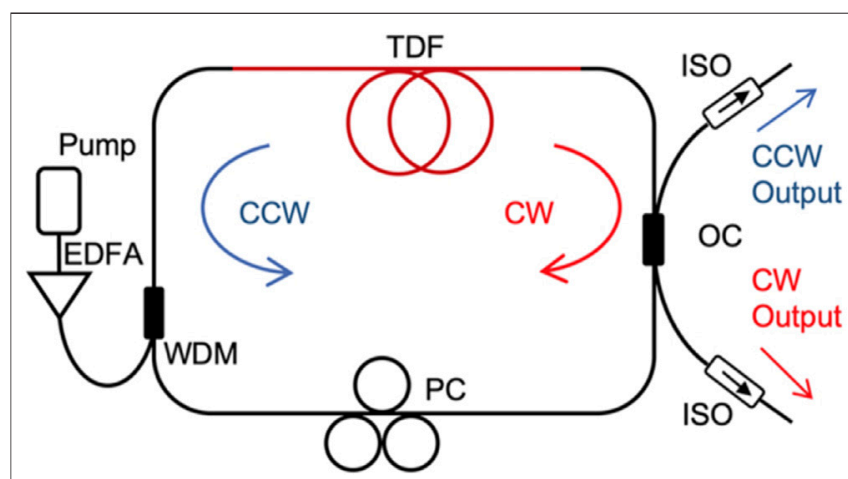

FIGURE 8 | Experimental setup of bidirectional Tm-doped selfsweeping fiber laser [23].

even it could be adjusted to zero to obtain a fixed wavelength output.

\section{Other Doped Self-Sweeping Fiber Lasers}

So far, self-sweeping effect has been observed not only in the most typical three bands of ytterbium-doped, erbium-doped, and thulium-doped fiber lasers, but also in different bands based on typical F-P linear cavities using other doped fibers as gain media.

In 2013, Pu Zhou's group at the National University of Defense Technology [34] first achieved the self-sweeping effect of thulium-holmium co-doped fiber lasers in the $1.9 \mu \mathrm{m}$ band with a self-sweeping range between 4 and $17 \mathrm{~nm}$ and a selfsweeping rate between $0.4 \mathrm{~nm} / \mathrm{s}$ and $1.5 \mathrm{~nm} / \mathrm{s}$. In 2015, Ref. [35] first investigated bismuth-doped self-sweeping fiber lasers in the $1,460 \mathrm{~nm}$ central wavelength band obtained a maximum selfsweeping range of $10 \mathrm{~nm}$ with a self-sweeping rate of $0.75 \mathrm{~nm} / \mathrm{s}$. In 2017, Peterka's group achieved and extended the self-sweeping band of holmium-doped self-sweeping fiber laser to about $2.1 \mu \mathrm{m}$ for the first time, obtaining self-sweeping ranges of about $4 \mathrm{~nm}$ [36] and $6 \mathrm{~nm}$ [37].

In 2021, Ref. [38] found that holmium-doped fibers are unstable in the self-sweeping state due to large pump absorption and large quantum defects between the pump and laser radiation that make the fiber heating uneven. Then they solved the fiber heating problem by using full bias-preserving elements and gaining fiber water cooling to obtain stable operation of the holmium-doped self-sweeping fiber laser. The laser achieves a stable self-scan of $10 \mathrm{~nm}$ in the $2.1 \mu \mathrm{m}$ band with a self-sweeping rate of $2-8 \mathrm{~nm} / \mathrm{s}$ and an average output power of more than $200 \mathrm{~mW}$. The sweeping range does not exceed $7 \mathrm{~nm}(2,108-2101 \mathrm{~nm})$ when the $7 \mathrm{~m}$ passive fiber is removed, or the maximum spectral range can be reduced to $7 \mathrm{~nm}$ by shortening the gain fiber to $0.8 \mathrm{~m}$. The first neodymium-doped self-sweeping fiber laser was realized by Lobach's group [39] in 2019. Two sections of bias-preserving fiber with lengths of 21 and $33 \mathrm{~cm}$ were fused in the resonator as Lyot filters for additional spectral selection. The laser obtained the self-sweeping effect near the 1.06 and $0.93 \mu \mathrm{m}$ bands, respectively. The self-scan spectra was tuned up to $1.8 \mathrm{~nm}$ and the self-sweeping rate was up to $9 \mathrm{~nm} / \mathrm{s}$. 
TABLE 1 | Summary of parametric studies of various doped self-sweeping fiber lasers.

\begin{tabular}{|c|c|c|c|c|c|}
\hline $\begin{array}{l}\text { Resonator gain } \\
\text { media }\end{array}$ & Sweeping direction & $\begin{array}{l}\text { Wavelength range } \\
\text { (nm) }\end{array}$ & $\begin{array}{l}\text { Sweeping range } \\
(\mathrm{nm})\end{array}$ & $\begin{array}{l}\text { Sweeping rate } \\
(\mathrm{nm} / \mathrm{s})\end{array}$ & References \\
\hline Linear (YDF) & Normal & $1,081-1,087$ & 7 (maximum) & -- & {$[14]$} \\
\hline Linear (YDF) & Normal & $1,062-1,078$ & $0.1-16$ & $0.5-16$ & [15] \\
\hline Linear (YDF) & Normal & $1,028-1,080$ & 30 (maximum) & -- & [19] \\
\hline Linear (YDF) & $\begin{array}{l}\text { Normal } \\
\text { Reverse }\end{array}$ & $\begin{array}{l}1,076-1,083 \\
1,073-1,079\end{array}$ & $\begin{array}{l}2.5-6.8 \\
4.3-5.3\end{array}$ & $\begin{array}{l}0.3-6.6 \\
0.5-1.7\end{array}$ & {$[22]$} \\
\hline Ring resonator (YDF) & Reverse & $\begin{array}{l}1,060.468-1,052.984 \\
1,070.448-1,066.026\end{array}$ & $\begin{array}{c}\sim 7.48 \\
--\end{array}$ & $\begin{array}{c}0.158-0.13 \\
0.0018-0.0002\end{array}$ & {$[25]$} \\
\hline Ring resonator (YDF) & Reverse & 1,055.6-1,034.6 & -- & $0.19-0.43$ & {$[26]$} \\
\hline Ring resonator (YDF) & Reverse & -- & $1.5-4.85$ & $0.145-0.284$ & [28] \\
\hline Linear (EDF) & $\begin{array}{l}\text { Normal } \\
\text { Reverse }\end{array}$ & $1,540-1,570$ & $\begin{array}{l}0.37 \\
0.43\end{array}$ & $\begin{array}{l}\sim 2.85 \\
\sim 1.48\end{array}$ & [29] \\
\hline Linear (EDF) & Reverse & 1,605 & $0.7-2.8$ & 0.05 (mimimum) & {$[30]$} \\
\hline Linear (TDF) & $\begin{array}{l}\text { Normal } \\
\text { Reverse } \\
\text { Wavelength stopping }\end{array}$ & 1920 & 26 (maximum) & -- & {$[31]$} \\
\hline Ring resonator (TDF) & Reverse & 1950 & $\sim 15$ & $\sim 0.2$ & {$[33]$} \\
\hline Linear (THDF) & Normal & 1900 & $4-17$ & $0.4-1.5$ & [34] \\
\hline Linear (BDF) & Normal & 1,460 & $\sim 10$ & $\sim 0.75$ & [35] \\
\hline Linear (HDF) & Normal & 2,100 & $\sim 10$ & 2-8 & [38] \\
\hline Linear (NDF) & Normal & $\begin{array}{c}1,060 \\
930\end{array}$ & $\begin{array}{c}1.8 \text { (maximum) } \\
\sim 0.2\end{array}$ & $\begin{array}{c}9 \text { (mimimum) } \\
--\end{array}$ & [39] \\
\hline
\end{tabular}

Table 1 summarizes the research results of the self-sweeping fiber lasers presented in Diversely Doped Self-Sweeping Fiber Lasers, and provides a comparative analysis of their selfsweeping range, self-sweeping rate, and self-sweeping direction. As can be seen from the table, the reported research results cover the self-scan band from near $1 \mu \mathrm{m}$ to near $2.1 \mu \mathrm{m}$, and the resonator structure involves both linear and ring resonators.

\section{PULSE TYPE OF SELF-SWEEPING FIBER LASER}

The pulse intensity dynamics of the self-sweeping effect is one of the distinguishing features of self-sweeping fiber lasers. Based on the reported self-sweeping literature, it is known that fiber lasers generate self-sweeping by forming dynamic gain or phase gratings in the gain fiber. Since there are few longitudinal modes in the laser output, the pulse sequence is modulated in an inter-mode beat frequency manner [40] and is associated with relaxation oscillations, which cause self-pulse effects [41] and exhibit microsecond pulse sequences. Currently, a variety of microsecond pulse sequences have been observed in doped self-sweeping fiber lasers, which can be broadly classified into the following four categories: typical microsecond pulse sequences, discontinuous pulse sequences, quasi-continuous pulse sequences, and novel pulse sequences.

\section{Typical Microsecond Pulse Sequence}

Microsecond pulse sequences are typical pulse intensity time domain dynamics of self-sweeping fiber lasers. Most of the doped (ytterbium-doped, thulium-doped, holmium-doped, thuliumholmium co-doped) self-sweeping fiber lasers are obtained with irregular microsecond pulse sequences, as shown in Figure 9A. Significant beat frequency modulation is observed after amplification of each pulse, as shown in the inset in Figure 9A. By limiting the number of longitudinal modes in the resonator by adjusting the laser resonator length, a singlefrequency self-sweeping fiber laser can be obtained. Compared with a self-sweeping fiber laser operating with multiple longitudinal modes, the microsecond pulse sequence obtained from a single-frequency self-sweeping fiber laser has a more pronounced periodic, regular nature. Figure 9B shows a typical microsecond pulse sequence of a single-frequency selfsweeping fiber laser [39]. Figure 9C shows its radio frequency spectrum (RF), which consists mainly of two peaks corresponding to the main (frequency near $0 \mathrm{MHz}$ ) mode and the nearest neighbor (frequency of $7.8 \mathrm{MHz}$ ) mode, respectively. In the RF spectrum, the intensity of the nearest neighbor mode is $10 \mathrm{~dB}$ smaller than that of the main mode. Based on these results, the researchers concluded that each pulse actually consists of a single longitudinal mode, which was also confirmed by using the delayed self-heterodyne method [42].

\section{Discontinuous Pulse Sequence}

In 2020, our group [25] observed the reverse self-sweeping effect in a bidirectional fiber ring resonator ytterbium-doped selfsweeping fiber laser, in which an ultra-slow self-sweeping operation was achieved with a 10/90 coupling ratio coupler in the resonator and a discontinuous pulse sequence was observed. Figure 10A shows the pulse intensity signal in the $30 \mathrm{~ms}$ range recorded at a pump power of $32 \mathrm{~mW}$, and a group of pulses can be observed near $1 \mathrm{~ms}$. The pulse intensity signal shows that the pulse signal is discontinuous under the ultra-slow self-scan operation. When this set of pulses is amplified, the average pulse repetition frequency of the microsecond pulse sequence 
A

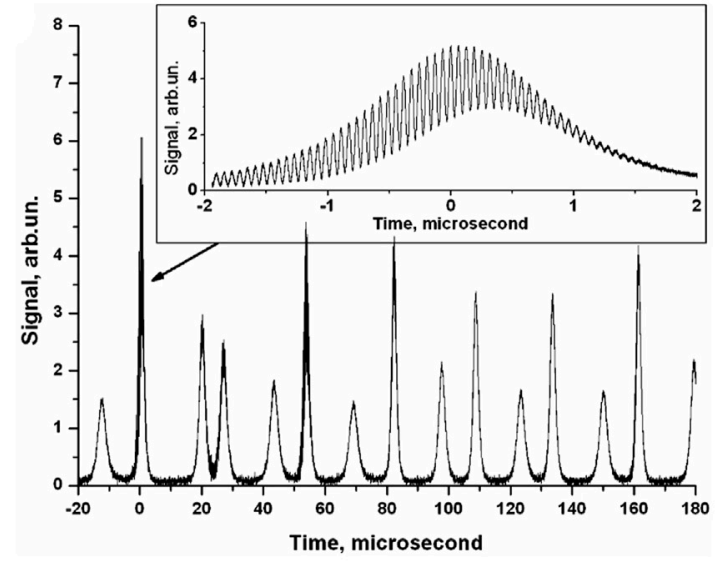

B

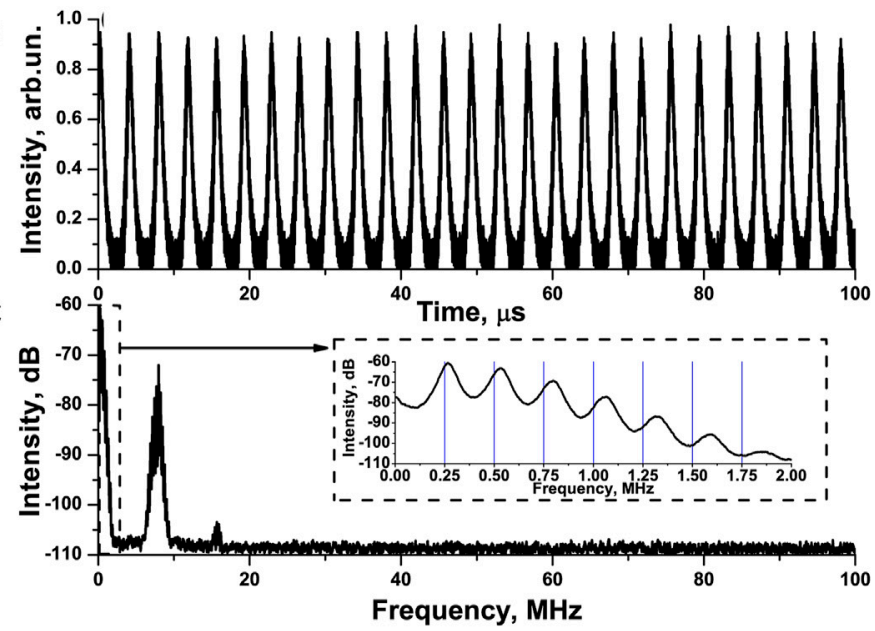

FIGURE 9 | (A) Typical irregular microsecond pulse sequences [15] (Inset) Beat modulation; (B) Typical single-frequency pulse sequences [39]; (C) RF spectrum of single-frequency self-sweeping fiber laser [39].

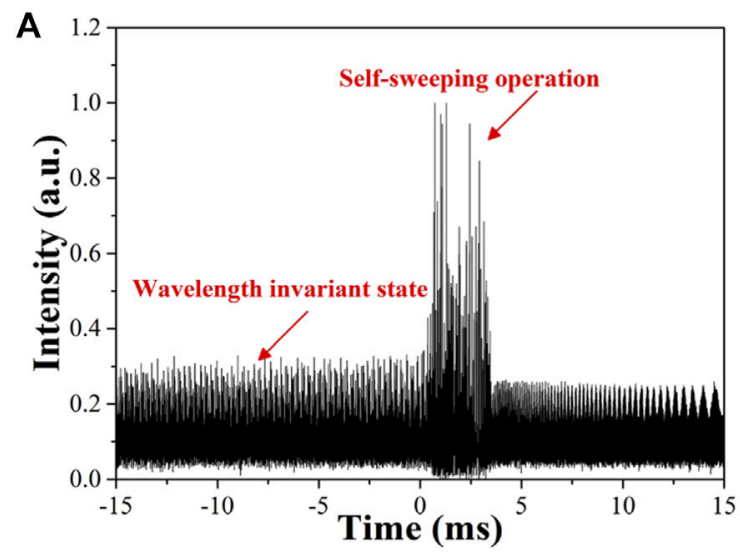

FIGURE 10 | Pulse intensity dynamics under ultra-slow self-sweeping operation [25]. pulse sequence in a magnified view within $1 \mathrm{~ms}$

is $28 \mathrm{kHz}$. At this point, the microsecond pulse signal in Figure 10B can be considered to represent the normal selfscan operation, and the rest of the pulse signals can be considered as wavelength-invariant states. Super-slow self-scan is also caused by large intervals (hundreds or tens of milliseconds) of microsecond pulse sets.

\section{Quasi-Continuous Pulse Sequence}

In 2020, Lobach's group [30] observed a novel pulse time domain dynamic in an erbium-doped self-sweeping fiber laser that obtained a continuous wave output with periodic bursts of intensity instead of the near-zero power microsecond pulse sequence between pulses of other self-sweeping fiber lasers, as shown in Figure 11A. This new pulse intensity dynamic consists of a pure sinusoidal waveform (Figure 11B) and a periodic pulse train (Figure 11C), and Fourier analysis of the pulse signal shows that the pulse train consists of two

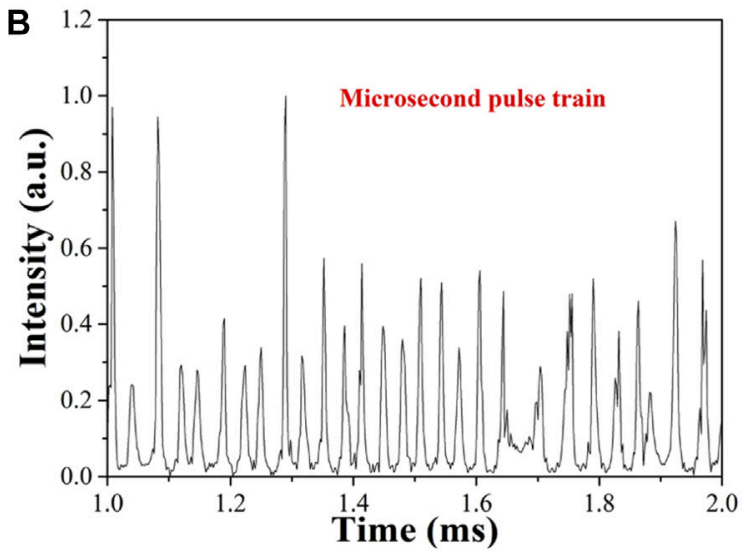

(A) Discontinuous pulse signal within $30 \mathrm{~ms}$; (B) A continuous microsecond longitudinal modes with an intermodal beat frequency modulation of 7.1 MHz, which manifests as a single peak in the RF spectrogram (Figure 11D). During the pulse generation, an additional peak at a multiplicative frequency appears in the RF spectrum (Figure 11E). On the other hand, the zero-level intensity at the sinusoidal minimum (Figure 11B) proves that the amplitudes of the individual modes are approximately equal. In addition, the average power magnitude over $1 \mu$ s is essentially the same (red line in Figure 11A), which is in marked contrast to the microsecond pulse sequences generated in other current self-sweeping fiber lasers.

\section{Novel Pulse Sequences}

In 2021, our group [28] observed a new sequence of self-pulse signals, similar to spike pulses, in a single-frequency ytterbiumdoped self-sweeping fiber laser based on a fiber saturable absorber, as shown in Figure 12A. The laser hops over modes of the 


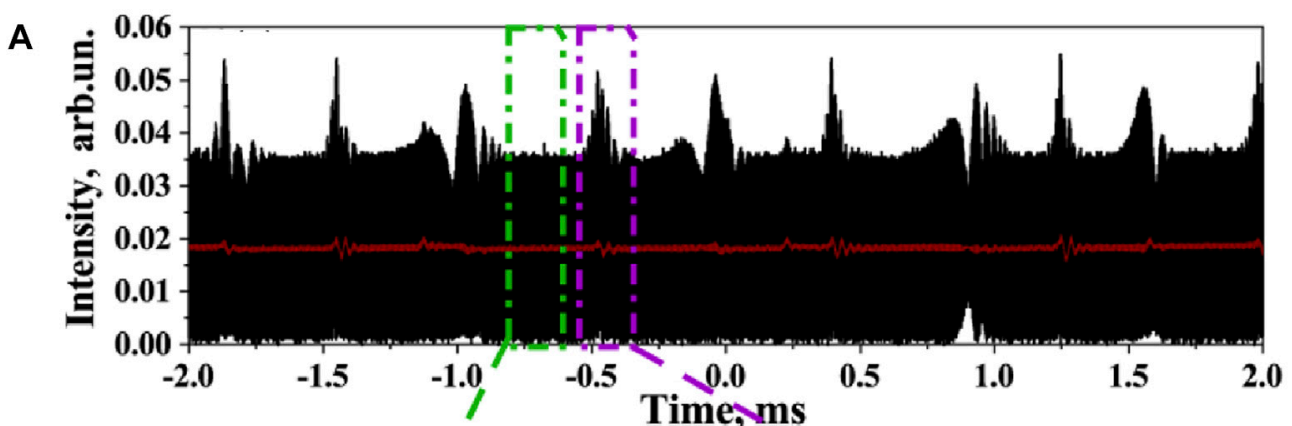

B

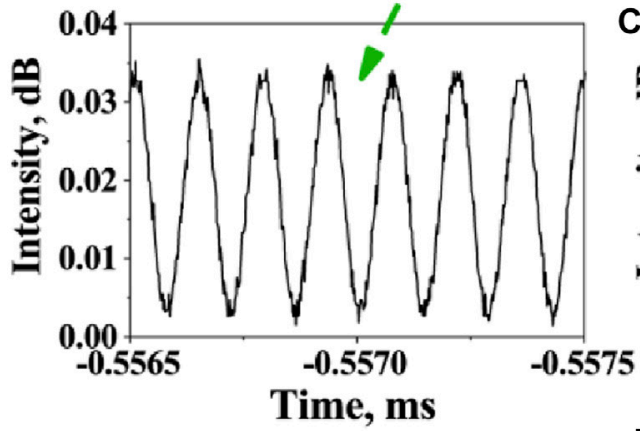

D

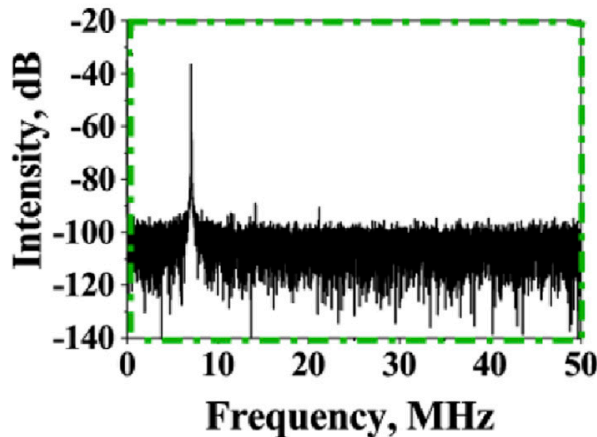

\section{c 0.06}

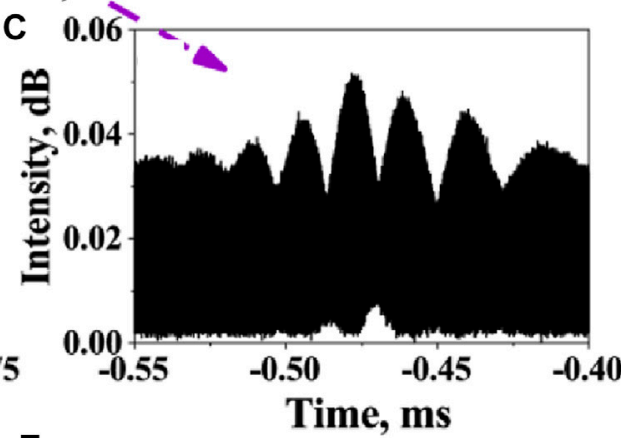

E

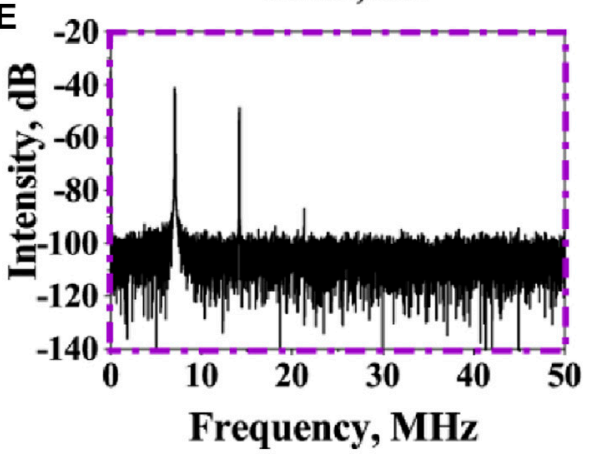

FIGURE 11 | Er-doped self-sweeping fiber laser [30]. (A) Overall pulse intensity dynamics; (B) Pure sinusoidal waveform intensity dynamics; (C) Periodic pulse string; (D) Left rectangular RF spectrum; (E) Right rectangular RF spectrum.

resonator. Time intervals of the single frequency operation are separated by short time intervals when the laser generates two neighbor lines (and old one and a new one) resulting in the sinusoidal beat signal. Figures 12B,C show the sinusoidal beat frequency modulation detail of $28.57 \mathrm{MHz}$ and a single beat frequency signal duration of $37.17 \mu$ s, respectively, as shown by the RF spectrum of the fiber laser in the inset of Figure 12C, which shows that the laser outputs two longitudinal modes during the duration. Moreover, it is clear that there are attenuation fluctuations at the dynamic end of the intensity, which we correspond with the simulation of the photon density variation of the fiber laser in Figure 12D.

\section{THE BASIC LAWS OF THE SELF-SWEEPING FIBER LASER}

The unique spectral dynamics as well as the pulse dynamics of the self-sweeping effect have been the focus of researchers since it was studied in the ytterbium-doped fiber laser in 2011. Under extensive experimental observations, researchers have demonstrated the dependence of the fundamental laws of selfsweeping such as self-sweeping range, average pulse repetition frequency and self-sweeping rate on pump power.

\section{Variation of Self-Sweeping Range}

From present reports, it is known that the variation of the output spectra of various doped self-sweeping fiber lasers with pump power or output power is roughly consistent. Figure 13A shows the variation of the self-sweeping range with output power for a typical self-sweeping fiber laser in a stable self-sweeping state [39], and its variation law with output power is similar to a parabolic function or e-exponential function. With the increase of pump power or output power, the self-sweeping range will gradually increase, and after reaching the maximum value, it will gradually decrease and then slowly disappear due to the interference of nonlinear effects such as the excited stimulated Brillouin scattering (SBS) effect [43] and SRS effect in the resonator. 

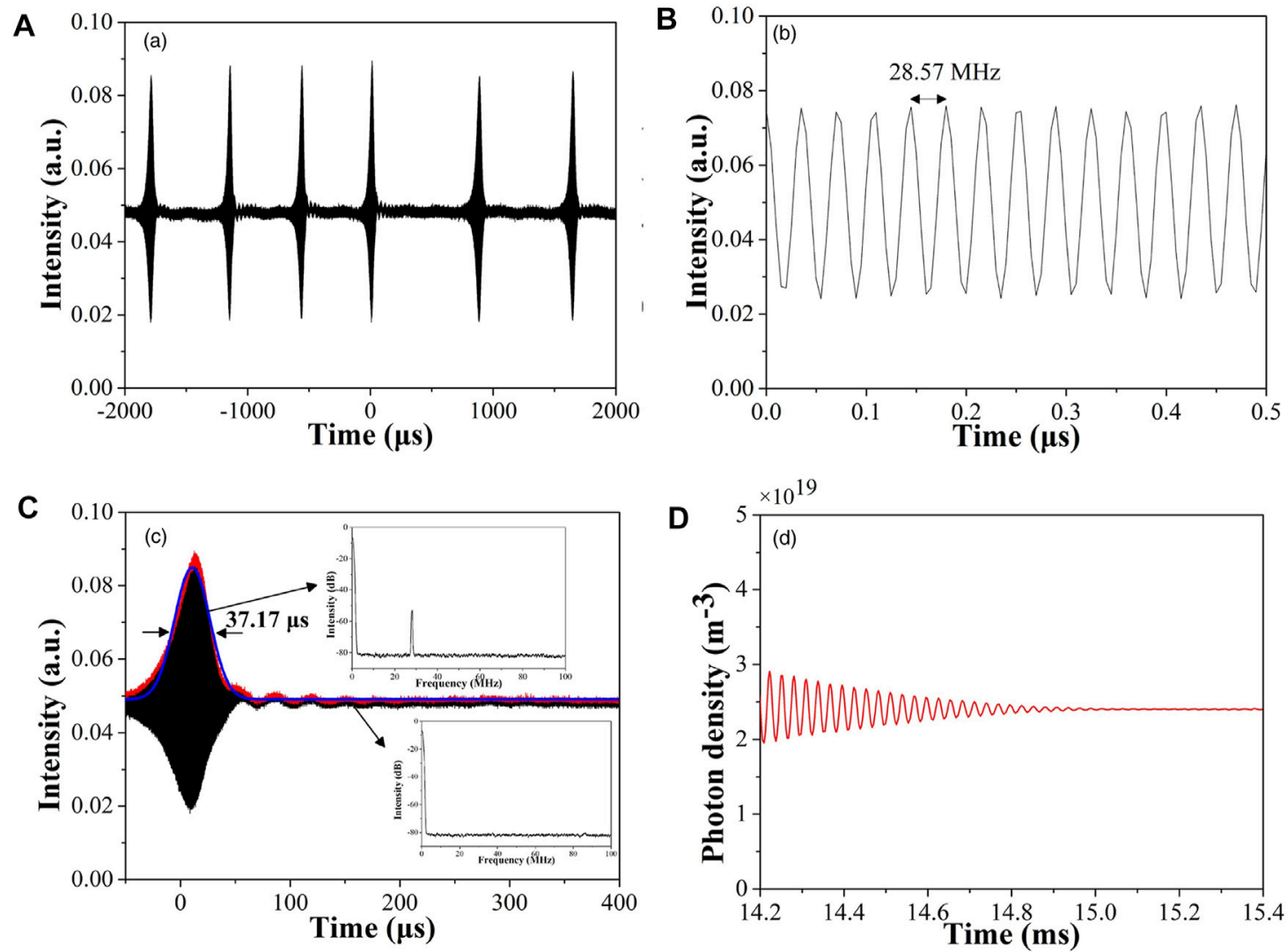

FIGURE 12 | Yb-doped self-sweeping fiber laser [28]. (A) Pulse intensity dynamics; (B) Pulse detail expansion; (C) Dynamic intensity of single pulse (inset) RF spectrum of pulse and continuous wave, (D) Simulated changes in photon density.
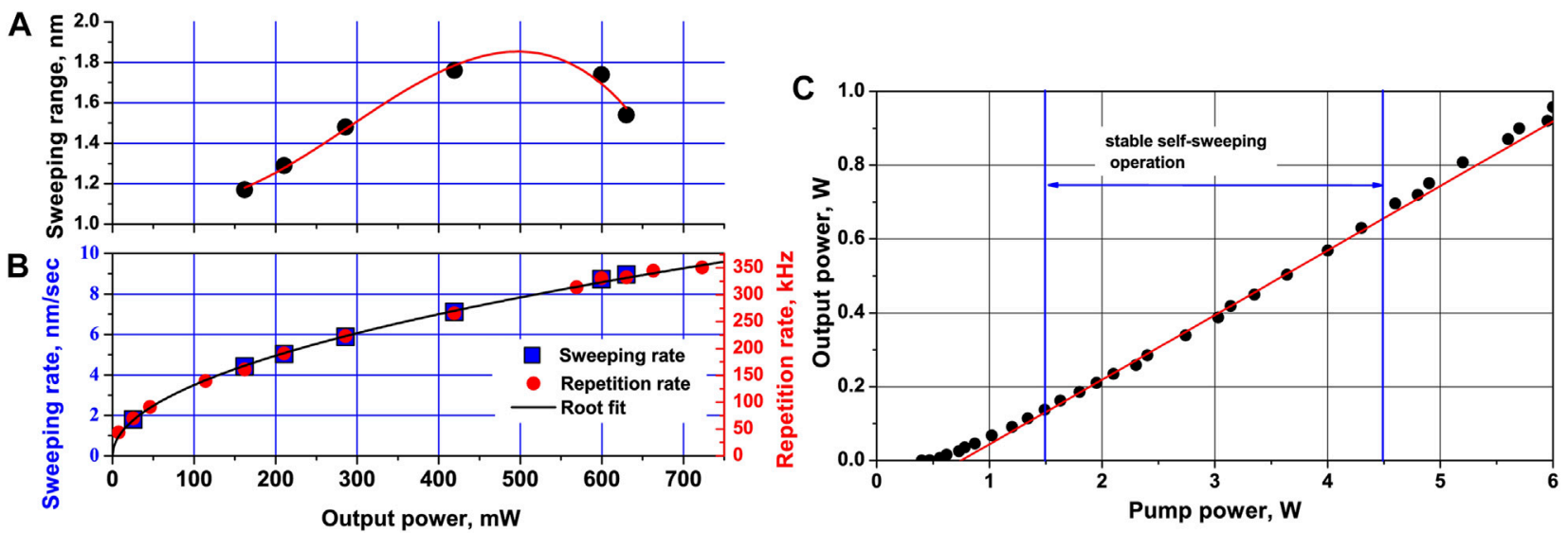

FIGURE 13 | Typical self-sweeping fiber laser [39] (A) Sweeping spectral range changes; (B) Variation of average pulse repetition frequency and sweeping rate; (C) Output slope efficiency.

\section{Variation of Average Pulse Repetition Frequency}

Figure 13B demonstrates that the average pulse repetition frequency of a typical self-sweeping fiber laser shows a specific pattern with pump power, i.e., the average pulse repetition frequency increases with increasing output power. Based on a large number of experimental studies, the researchers concluded that the average pulse repetition frequency is linearly related to the squared output power [39], following the Equation: 


$$
\Delta v=a \sqrt{P}
$$

Where: $\Delta v$ is the average pulse repetition frequency; $a$ is the scale factor; $P$ is the output power.

From the reported self-sweeping literature, it is clear that the relationship between the average pulse repetition frequency and output power follows Eq. 1, regardless of whether it is a typical microsecond pulse sequence, a discontinuous pulse signal, a quasi-continuous pulse signal or a novel pulse signal.

\section{Variation of Self-Sweeping Rate}

The self-sweeping rate depends on the speed of dynamic grating generation and removal in the gain medium, a grating generation and removal process represents a self-sweeping cycle, if the selfthis cycle is shortened, then the self-sweeping rate will naturally become faster. The relationship between the self-sweeping rate and the variation of pump power or output power satisfies the following equation:

$$
V=b \sqrt{P}
$$

Where: $V$ is the self-sweeping rate; $b$ is the scale factor; $P$ is the output power.

This equation is in clear agreement with Eq. 1 for the variation of the average pulse repetition frequency with pump power or output power. This indicates that the self-sweeping rate is consistent with the average pulse repetition frequency, i.e., both increase with the increase of pump power or output power. Figure 13B shows a graph of the variation of the selfsweeping rate with output power for a typical self-sweeping fiber laser [39], following Eq. 2 above.

\section{Pump Power Range of Self-Sweeping Effect}

Based on the reported self-sweeping fiber laser, it is known that the self-sweeping effect does not generally occur directly at the laser threshold, but is generated and continues to operate for a period of time at some power range above the laser threshold. Figure 13C shows the output slope efficiency of a typical selfsweeping fiber laser [39], where the self-sweeping effect can be seen to occur between 1.5 and $4.5 \mathrm{~W}$ above the pump power threshold. The reason for its appearance only in a certain power range and its disappearance after a certain period of time is that the stable conditions for the formation of self-sweeping are destroyed by nonlinear effects such as SBS effect and SRS effect in the resonator.

\section{APPLICATIONS OF THE SELF-SWEEPING FIBER LASER}

\section{Spectral Detection}

The laser output wavelength with stable periodic spontaneous tuning is a distinctive feature of the self-sweeping fiber laser, and the detection by high-resolution interferometry shows that the jump interval of the self-sweeping fiber laser wavelength is very narrow. Therefore, the self-sweeping fiber laser can be used for the spectral detection of wide and narrow band FBGs as well as bandpass filters and other filter devices.
In 2013, Lobach's group [17] conducted the first study related to the application of self-sweeping fiber lasers. This experiment used an ytterbium doped self-sweeping fiber laser to measure the reflection spectrum of a $\pi$-phase-shifted grating [44]. The reflectance spectra of the phase-shifted grating in the wider and narrower band ranges measured using this method are in good agreement with the reflectance spectra from standard measurements. This experiment demonstrates that the accuracy of the self-sweeping fiber laser is reliable for narrow-band and broad-band reflection spectra detection. Later, in 2018, the group [31] used a thulium-doped self-sweeping fiber laser as a light source to measure the absorption spectra of water molecules in air. Figure 14 shows that the experimentally measured absorption spectra of water molecules in air agree well with the actual absorption spectra. Where the small differences observed between experiments and simulations around the intensity maximum (about 1) are related to the weak power modulation of the self-sweeping fiber laser.

The holmium doped self-sweeping fiber laser near the $2.06 \mu \mathrm{m}$ band studied by the group [45] in 2020 can be used for the spectral detection and analysis of ${ }^{12} \mathrm{CO}_{2}$ and ${ }^{13} \mathrm{CO}_{2}$ isotopes. The holmium-doped self-sweeping fiber laser near the $2.1 \mu \mathrm{m}$ band studied by the group [38] in 2021 has absorption lines in the spectral region of $\mathrm{N}_{2} \mathrm{O}$ and can be used for its spectral detection. All these works show that self-sweeping fiber lasers are more reliable for spectral detection applications.

\section{Short-Pulse Synthesis}

A new technique for short pulse synthesis in the Fourier domain was reported by Lobach's group [46] in 2015, as shown in Figure 15. The synthesis method differs from the conventional intracavity mode-locking of multimode lasers [47, 48], Fourierdomain mode-locking of tunable lasers [49], Fourier synthesis of multiple independent sources [50,51], and line-by-line processing [52]. This new technique uses a single-frequency ytterbium-doped self-sweeping fiber laser instead of multiple independent sources to extract a single resonator mode as an entire time series and to perform coherent synthesis in an external circumferential resonator. This experiment allows the combination of 20 singlemode pulses to achieve the synthesis of different waveforms, but the disadvantage is that the pulse intensity fluctuations of the selfsweeping fiber laser can cause mode-limitation.

\section{Optical Fiber Sensing}

Due to the lack of reflection sensitivity of fiber optic sensor devices designed based on self-sweeping light sources, the use of such devices for any sensing application is limited, and this problem is now generally solved by using FBGs with externally induced modulation of refractive index to increase the reflection signal strength. In 2016, the team of Lobach [20] designed a fiber optic sensing interrogator using an ytterbium-doped self-sweeping fiber laser, which was successfully tested on a sensing line consisting of six FBG sensors, achieving an accuracy estimate of $\sim 600 \mathrm{MHz}(2$ $\mathrm{pm})$ spectral measurements, comparable to actual measurement instruments. In addition, the device has a higher spectral resolution and higher peak output power than comparable devices, which means that multiple sensor sub-lines can operate simultaneously. In 2020, the group [53] realized the application of a frequency 


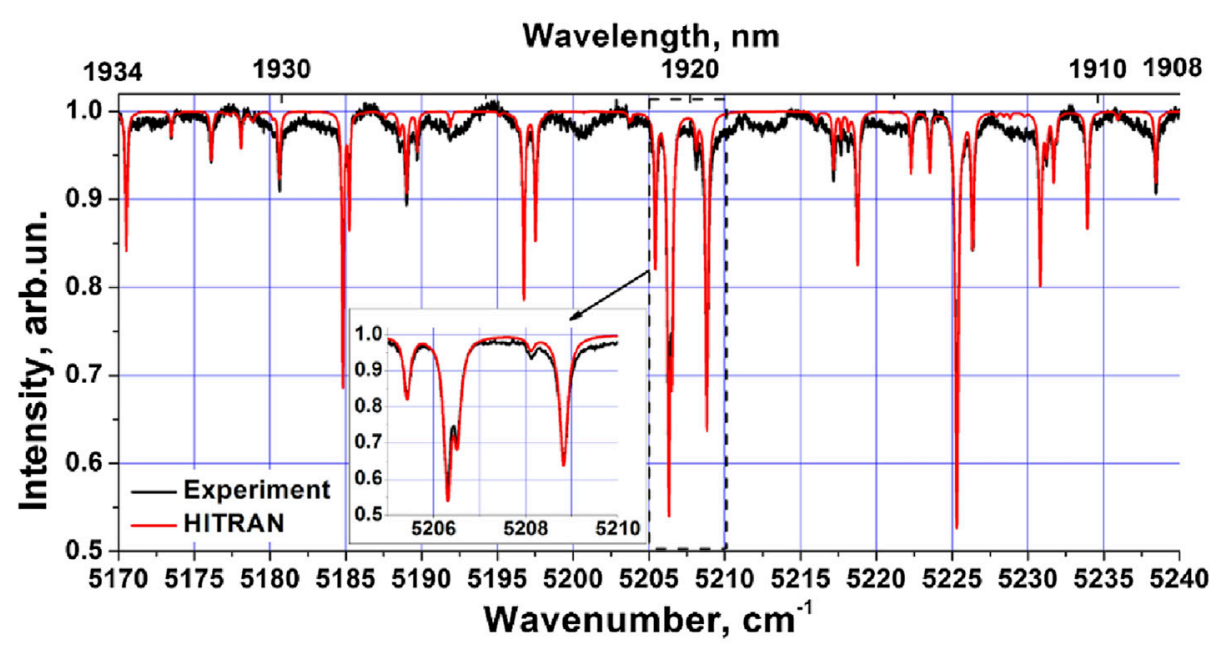

FIGURE 14 | Measurement of absorption spectra of water molecules by Tm-doped self-sweeping fiber laser [31].
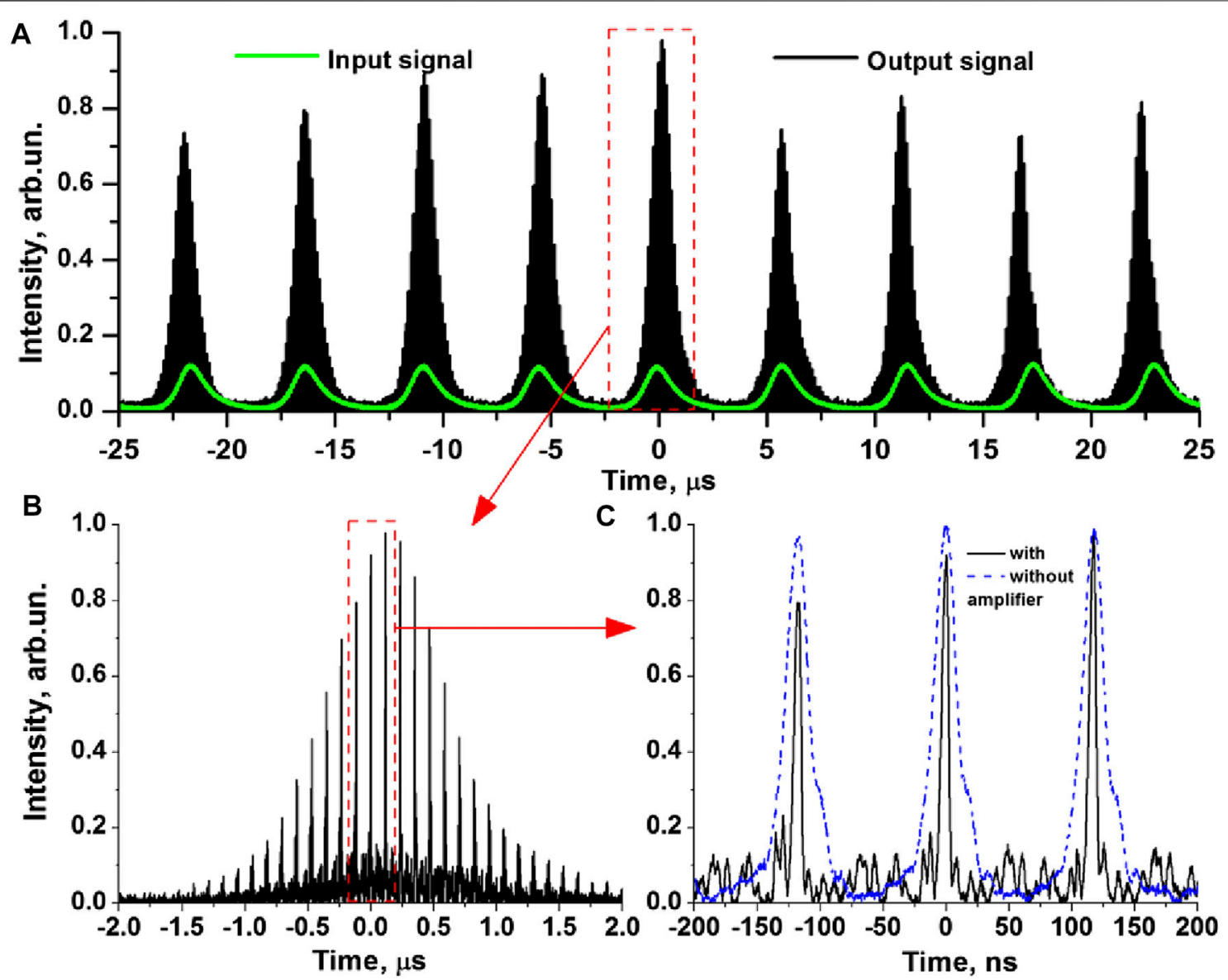

FIGURE 15 | (A) Output synthesis pulse [46]; (B) Envelope of synthesis pulse; (C) Single pulse.

domain reflectometer based on a self-sweeping laser for sensing. As shown in Figure 16, a sensing line was fused to one interferometer arm of the Mach-Zehnder interferometer, and a set of FBG arrays were used on the sensing line, which consisted of one reference FBG (1,064 nm) of a Bragg wavelength inside the sweeping range of the laser and 28 equidistant FBGs $(1,092 \mathrm{~nm})$ of Bragg 


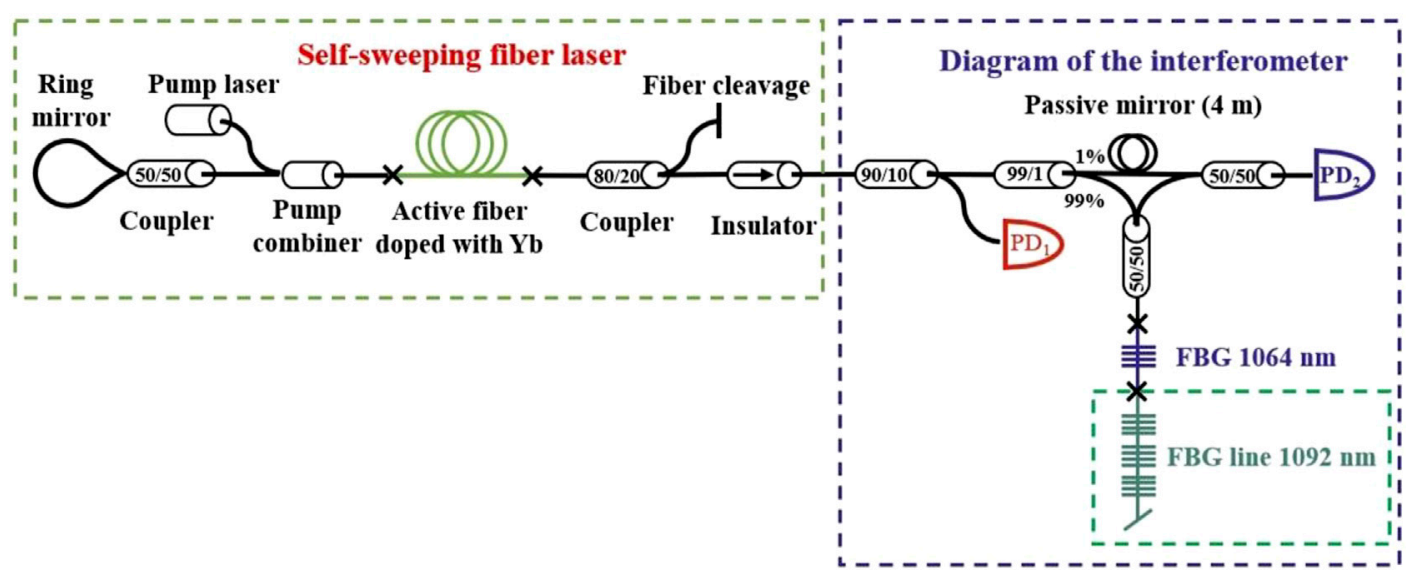

FIGURE 16 | The experimental device of the frequency-domain reflectometer consisting of a self-sweeping laser and a Mach-Zehnder interferometer: (PD1, PD2) photodiodes [53].

wavelength outside (but close to) the sweeping range. Each time domain pulse generated by this self-sweeping fiber laser corresponds to a specific frequency in the spectral region. The frequency change interval is $5.5 \mathrm{MHz}$ in one scan cycle, and the pulses are equally spaced in the frequency domain. The longitudinal distribution of the reflected signal on the fiber was obtained by analyzing the interferometric reflection signal dependent on the optical frequency or the number of pulses by means of the fast Fourier transform. Each peak on the reflection map corresponds to an FBG reflection signal, and since the FBG in the $1,064 \mathrm{~nm}$ band is in the self-sweeping tuning range, its reflection signal has a higher amplitude compared to the FBG at $1,092 \mathrm{~nm}$, which is not in the tuning range. The reflection spectra of individual FBGs can be obtained by selectively analyzing a region through Fourier inversion. Since the reflection spectrum of the 1,092 nm FBG has a narrower peak, a temperature change can be more clearly observed in the peak shift values, so this approach may be useful for temperature measurements. In fact, tunable lasers using special devices have sweeping ranges of more than $100 \mathrm{~nm}$ and sweeping rates of up to a million nanometers per second [54]. Also, sensor systems for practical applications usually require a sweeping range of $40 \mathrm{~nm}$ and a sweeping rate of no less than $10 \mathrm{~nm} / \mathrm{s}$, which is not yet met by sensor systems using selfsweeping light sources, which achieve a suboptimal spectral range. Most fiber grating sensors on the market operate in the $1.5 \mu \mathrm{m}$ spectral range, while erbium-doped self-sweeping fiber lasers operating near $1.5 \mu \mathrm{m}$ have a small sweeping range of $2.8 \mathrm{~nm}$, which greatly limits their practical applications.

Now that a decade has passed since the development of the self-sweeping fiber laser, many practical applications are still waiting to be explored by researchers. We believe that in addition to spectral detection, short pulse synthesis and fiber sensing, there are more potential applications for self-sweeping fiber lasers in fiber optic communication and coherent synthesis. With further research on self-sweeping fiber lasers, they will be more widely used and their development will continue to open up a new chapter.

\section{SUMMARY AND OUTLOOK}

This paper reviews the research progress of various doped selfsweeping fiber lasers in the last decade, including the spectral range, fundamental laws, dynamic types of pulse intensity, and recent application progress of self-sweeping fiber lasers. The currently reported self-sweeping fiber lasers are capable of generating autonomous wavelength tuning in different spectral ranges between 1 and $2.1 \mu \mathrm{m}$, and related application studies are also conducted in spectral detection and analysis, and optical sensing applications. However, the current research on self-sweeping fiber lasers is still at the initial stage, and the principle of the selfsweeping effect needs to be explained more comprehensively and profoundly. Establishing a theoretical model to predict the specific behavioral dynamics of self-sweeping considering all influencing factors is the direction researchers need to work on. Among them, precise observation of small wavelength drifts and expansion of more wavelength bands and wider self-sweeping range are also the goals pursued by researchers. In the application of self-sweeping fiber laser, firstly, the laser needs to be packaged and miniaturized for practical application, and secondly, considering that the selfsweeping fiber laser is influenced by the environment and resonator loss, the temperature and resonator loss control module needs to be added to stabilize the self-scanning range, and the spectral selection device needs to be added to reduce the boundary fluctuation of the scanning area, and the self-sweeping range needs to be artificially regulated. The current applications of self-sweeping fiber lasers are mainly in the field of spectroscopy, and a lot of research needs to be done to expand the field of fiber sensing and communication.

\section{DATA AVAILABILITY STATEMENT}

The original contributions presented in the study are included in the article/Supplementary Material, further inquiries can be directed to the corresponding authors. 


\section{AUTHOR CONTRIBUTIONS}

$\mathrm{ZZ}$ and $\mathrm{BL}$ finished writing the whole article. $\mathrm{KW}$ is responsible for part of the work. $\mathrm{HC}$ and JB is responsible for article modification.

\section{REFERENCES}

1. Villatoro J, Monzon-Hernandez D. Low-cost Optical Fiber Refractive-index Sensor Based on Core Diameter Mismatch. J Lightwave Technol (2006) 24(3): 1409-13. doi:10.1109/JLT.2005.863246

2. Xia T-H, Zhang AP, Gu B, Zhu J-J. Fiber-optic Refractive-index Sensors Based on Transmissive and Reflective Thin-Core Fiber Modal Interferometers. Opt Commun (2010) 283(10):2136-9. doi:10.1016/j.optcom.2010.01.031

3. Qiao S, Ma Y, He Y, Patimisco P, Sampaolo A, Spagnolo V. Ppt Level Carbon Monoxide Detection Based on Light-Induced Thermoelastic Spectroscopy Exploring Custom Quartz Tuning forks and a Mid-infrared QCL. Opt Express (2021) 29(16):25100-8. doi:10.1364/OE.434128

4. Lang Z, Qiao S, He Y, Ma Y. Quartz Tuning fork-based Demodulation of an Acoustic Signal Induced by Photo-Thermo-Elastic Energy Conversion. Photoacoustics (2021) 22:100272. doi:10.1016/j.pacs.2021.100272

5. Dong B, Wei L, Zhou D-P, Liu W-K, Lit JWY. Core-offset Small-CoreDiameter Dispersion Compensation Fiber Interferometer and its Applications in Fiber Sensors. Appl Opt (2009) 48(23):4577-81. doi:10.1364/AO.48.004577

6. Yang Z, Wu X, Ouyang D, Sun H, Ruan S. Analysis of Performance Improvement in a Swept Source for Optical Coherence Tomography Applications. Jpn J Appl Phys (2020) 59(3):030904. doi:10.35848/1347-4065/ ab7216

7. Fukushima K, Bui QH, Nakaya K, Soares MG, Wada A, Tanaka S. EDF Ring Laser Using Cascaded-Chirped Long Period Fiber Grating for Temperature Measurement. Opt Express (2020) 28(9):13081-90. doi:10.1364/OE.389235

8. Woodward RI, Majewski MR, Hudson DD, Jackson SD. Swept-wavelength Mid-infrared Fiber Laser for Real-Time Ammonia Gas Sensing. APL Photon (2019) 4(2):020801. doi:10.1063/1.5065415

9. Sarmani AR, Sheih S-J, Mahamd Adikan FR, Mahdi AM. Spectral Hole Burning Effects Initiated by Uniform Signal Intensities in a Gain-Flattened EDFA. Chin Opt Lett(2011) 9(2):020603-6. doi:10.3788/COL201109.020603

10. Peterka P, Honzátko P, Koška P, Todorov F, Aubrecht J, Podrazký O. Reflectivity of Transient Bragg Reflection Gratings in Fiber Laser with Laser-Wavelength Self-Sweeping: Erratum. Opt Express (2016) 24(14): 16221. doi:10.1364/oe.24.016221

11. Peterka P, Honzátko P, Koška P, Todorov F, Aubrecht J, Podrazký O. Reflectivity of Transient Bragg Reflection Gratings in Fiber Laser with Laser-Wavelength Self-Sweeping. Opt Express (2014) 22(24):30024. doi:10.1364/OE.22.030024

12. Peterka P, Koska P, Ctyroky J. Reflectivity of Superimposed Bragg Gratings Induced by Longitudinal Mode Instabilities in Fiber Lasers. IEEE J Select Top Quan Electron. (2018) 24:1-8. doi:10.1109/JSTQE.2018.2806084

13. Hughes TP, Young KM. Mode Sequences in Ruby Laser Emission. Nature (1962) 196(4852):332-4. doi:10.1038/196332a0

14. Kir'yanov AV, Il'ichev NN. Self-induced Laser Line Sweeping in an Ytterbium Fiber Laser with Non-resonant Fabry-Perot Cavity. Laser Phys Lett (2011) 8(4): 305-12. doi:10.1002/lapl.201010138

15. Lobach IA, Kablukov SI, Podivilov EV, Babin SA. Broad-range Self-Sweeping of a Narrow-Line Self-Pulsing Yb-Doped Fiber Laser. Opt Express (2011) 19(18):17632-40. doi:10.1364/OE.19.017632

16. Lobach IA, Babin SI, Podivilov EV, Kablukov SA. All-fiber Broad-Range SelfSweeping Yb-Doped Fiber Laser. Proc SPIE-The Int Soc Opt Eng (2012) 8237: 30. doi:10.1117/12.906660

17. Lobach IA, Kablukov SI. Application of a Self-Sweeping Yb-Doped Fiber Laser for High-Resolution Characterization of Phase-Shifted FBGs. J Lightwave Technol (2013) 31(18):2982-7. doi:10.1109/JLT.2013.2278177

18. Navratil P, Peterka P, Kubecek V, Kalli K, Kanka J, Mendez A. Effect of Pump Wavelength on Self-Induced Laser Line Sweeping in Yb-Doped Fiber Laser.

\section{FUNDING}

National Natural Science Foundation of China (61905193); Key $\mathrm{R} \& \mathrm{D}$ project of Shaanxi Province-International Science and Technology Cooperation Programme (2020KW-018).

Micro-structured Specialty Opt Fibres (2013) 8775:87750D. doi:10.1117/ 12.2017471

19. Lobach IA, Yu Tkachenko A, Kablukov SI. Optimization and Control of the Sweeping Range in an Yb-Doped Self-Sweeping Fiber Laser. Laser Phys Lett (2016) 13(4):045104. doi:10.1088/1612-2011/13/4/045104

20. Tkachenko AY, Lobach IA, Guskov LN. Fiber Sensor Interrogator Based on Self-Sweeping Fiber Laser. Proceeding of the 2016 17th International Conference of Young Specialists on Micro/Nanotechnologies and Electron Devices (EDM), 30 June-4 July 2016, Erlagol, Russia, IEEE (2016) 348-51. doi:10.1109/EDM.2016.7538756

21. Tkachenko AY, Lobach IA, Kablukov SI. All-fiber Brillouin Optical Spectrum Analyzer Based on Self-Sweeping Fiber Laser. Opt Express (2017) 25(15): 17600. doi:10.1364/OE.25.017600

22. Navratil P, Peterka P, Honzatko P, Kubecek V. Reverse Spontaneous Laser Line Sweeping in Ytterbium Fiber Laser. Laser Phys Lett (2017) 14(3):035102 doi:10.1088/1612-202X/aa548d

23. Jiang H, Lei J, Set SY, Yamashita S. Spontaneous Laser Line Sweeping in Tm Doped Fiber Laser. Laser Congress 2018 (ASSL) (2018) 34. doi:10.1364/ ASSL.2018.AM6A.34

24. Wang K, Wen Z, Lu B, Qi X, Bai J. Wavelength Self-Sweeping Yb-Doped Bidirectional Fiber Ring Laser[C]. Proceeding of the Eleventh International Conference on Information Optics and Photonics (CIOP 2019); Xi'an, China (2019). doi:10.1117/12.2548390

25. Wang K, Wen Z, Chen H, Qi X, Lu B, Bai J. Observation of Reverse SelfSweeping Effect in an All-Polarization-Maintaining Bidirectional YtterbiumDoped Fiber Laser. Opt Express (2020) 28(9):13913-20. doi:10.1364/ OE.392903

26. Wang K, Wen Z, Chen H, Lu B, Bai J. Wavelength-flexible All-PolarizationMaintaining Self-Sweeping Fiber Laser Based on Intracavity Loss Tuning. Chin Opt Lett (2021) 19(4):041401. doi:10.3788/COL202119.041401

27. Stepanov S, Fotiadi A, Mégret P. Effective Recording of Dynamic Phase Gratings in Yb-Doped Fibers with Saturable Absorption at 1064nm. Opt Express (2007) 15(14):8832-7. doi:10.1364/OE.15.008832

28. Wen Z, Wang K, Chen H, Lu B, Bai J. Self-sweeping Ytterbium-Doped Fiber Laser Based on a Fiber Saturable Absorber. Appl Phys Express (2020) 14(1): 012005. doi:10.35848/1882-0786/abd37d

29. Navratil P, Peterka P, Vojtisek P, Kasik I, Aubrecht J, Honzatko P. Self-swept Erbium Fiber Laser Around $1.56 \mu \mathrm{m}$. Opto-Electronics Rev (2018) 26(1):29-34 doi:10.1016/j.opelre.2017.11.004

30. Kashirina EK, Lobach IA, Kablukov SI. Dual-longitudinal-mode CW SelfSweeping Operation in Er-Doped Fiber Laser. Opt Lett (2020) 45(24):6659-62. doi:10.1364/OL.412781

31. Budarnykh AE, Vladimirskaya AD, Lobach IA, Kablukov SI. Broad-range SelfSweeping Single-Frequency Linearly Polarized Tm-Doped Fiber Laser. Opt Lett (2018) 43(21):5307-10. doi:10.1364/OL.43.005307

32. Budarnykh AE, Lobach IA, Kablukov SI. Self-sweeping Tm-Doped Fiber Laser with Wavelength Stopping. Laser Phys Lett (2019) 16(2):025108. doi:10.1088/ 1612-202X/aaf804

33. Jiang H, Zhao Z, Jin L, Set SY, Yamashita S. Self-wavelength-sweeping in a Bidirectional Thulium-Doped Fiber Ring Laser. Appl Phys Express (2019) 12(4): 042006. doi:10.7567/1882-0786/ab071e

34. Wang X, Zhou P, Wang X, Xiao H, Si L. Tm-Ho Co-doped All-Fiber BrandRange Self-Sweeping Laser Around $19 \mu \mathrm{m}$. Opt Express (2013) 21(14):16290 doi:10.1364/OE 21.016290

35. Lobach IA, Kablukov SI, Melkumov MA, Khopin VF, Babin SA, Dianov EM. Single-frequency Bismuth-Doped Fiber Laser with Quasi-Continuous SelfSweeping. Opt Express (2015) 23(19):24833-42. doi:10.1364/OE.23.024833

36. Aubrecht J, Peterka P, Koška P, Podrazký O, Todorov F, Honzátko P. Selfswept Holmium Fiber Laser Near 2100 Nm. Opt Express (2017) 25(4):4120-5. doi:10.1364/oe.25.004120 
37. Aubrecht J, Peterka $\mathrm{P}$, Koška $\mathrm{P}$, Honzátko $\mathrm{P}$, Jelínek M, Kamrádek M. Spontaneous Laser-Line Sweeping in Ho-Doped Fiber Laser. Fiber Lasers XIV: Tech Syst (2017) 10083:100831V. doi:10.1117/12.2249486

38. Vladimirskaya AD, Kamynin VA, Lobach IA, Kablukov SI. Robust Operation of Linearly Polarized Broad-Range Self-Sweeping Ho-Doped Fiber Laser. Laser Phys Lett (2021) 18(7):075101. doi:10.1088/1612-202X/ac0154

39. Kashirina EK, Lobach IA, Kablukov SI. Single-frequency Self-Sweeping Nd-Doped Fiber Laser. Opt Lett (2019) 44(9):2252. doi:10.1364/ OL.44.002252

40. Fernandez Casares S, Balle S, Menendez-Valdes P. Mode Beating and Spontaneous Emission Noise Effects in a Variable-Waveguide Model for the Dynamics of Gain-Guided Semiconductor Laser Arrays. IEEE J Quan Electron. (1994) 30(11):2449-57. doi:10.1109/3.333695

41. Guan W, Marciante JR. Complete Elimination of Self-Pulsations in Dual-Clad Ytterbium-Doped Fiber Lasers at All Pumping Levels. Opt Lett (2009) 34(6): 815-7. doi:10.1364/OL.34.000815

42. Ludvigsen $H$, Tossavainen M, Kaivola M. Laser Linewidth Measurements Using Self-Homodyne Detection with Short Delay[J]. Opt Commun (1998) 155(1-3):180-6. doi:10.1016/S0030-4018(98)00355-1

43. Kobyakov A, Sauer M, Chowdhury D. Stimulated Brillouin Scattering in Optical Fibers. Adv Opt Photon (2010) 2(1):1-59. doi:10.1364/AOP.2.000001

44. Malara P, Campanella CE, De Leonardis F, Giorgini A, Avino S, Passaro VMN. Enhanced Spectral Response of $\pi$-phase Shifted Fiber Bragg Gratings in Closed-Loop Configuration. Opt Lett (2015) 40(9):2124-6. doi:10.1364/ OL.40.002124

45. Vladimirskaya AD, Skvorsov MI, Wolf AA, Kaminin VA, Lobach IA, Kablukov SI. Ho-doped Fiber Laser with Wavelength Self-Sweeping Near $2.06 \mu \mathrm{m}$ for CO2 Spectroscopy[C]. Proceeding of the International Conference Laser Optics (ICLO); Nov. 2020; St. Petersburg, Russia. IEEE (2020). doi:10.1109/iclo48556.2020.9285573

46. Lobach IA, Kablukov SI, Podivilov EV, Fotiadi AA, Babin SA. Fourier Synthesis with Single-Mode Pulses from a Multimode Laser. Opt Lett (2015) 40(15):3671-4. doi:10.1364/ol.40.003671

47. Song Y-W, Morimune K, Set SY, Yamashita S. Polarization Insensitive AllFiber Mode-Lockers Functioned by Carbon Nanotubes Deposited onto Tapered Fibers. Appl Phys Lett (2007) 90(2):021101. doi:10.1063/1.2431445

48. Song Y-W, Yamashita S, Goh CS, Set SY. Carbon Nanotube Mode Lockers with Enhanced Nonlinearity via Evanescent Field Interaction in D-Shaped Fibers. Opt Lett (2007) 32(2):148-50. doi:10.1364/OL.32.000148
49. Todor S, Biedermann B, Wieser W, Huber R, Jirauschek C. Instantaneous Lineshape Analysis of Fourier Domain Mode-Locked Lasers. Opt Express (2011) 19(9):8802. doi:10.1364/OE.19.008802

50. Hyodo M, Abedin KS, Onodera N. Generation of Arbitrary Optical Waveforms by Fourier Synthesis Using Three Continuous-Wave Semiconductor Lasers. Electron Lett (2000) 36(3):224-5. doi:10.1049/el: 20000212

51. Hyodo M, Onodera N, Abedin KS. Fourier Synthesis of 96-GHz Optical-Pulse Trains by Phase Locking of Three Continuous-Wave Semiconductor Lasers. Opt Lett (1999) 24(5):303. doi:10.1364/OL.24.000303

52. Zhao C, Deng W, Yan Y, Yao X. Progressive Line Processing of Kernel RX Anomaly Detection Algorithm for Hyperspectral Imagery. Sensors (2017) 17(8):1815. doi:10.3390/s17081815

53. Tkachenko AY, Smolyaninov NN, Skvortsov MI, Lobach IA, Kablukov SI. A Coherent Optical Frequency-Domain Reflectometer Based on a Self-Sweeping Fiber Laser for Sensing Applications. Instrum Exp Tech (2020) 63(4):532-7. doi:10.1134/S0020441220040338

54. Yamashita S, Takubo Y. Wide and Fast Wavelength-Swept Fiber Lasers Based on Dispersion Tuning and Their Application to Optical Coherence Tomography. Photonic Sens (2013) 3(4):320-31. doi:10.1007/s13320-0130129-0

Conflict of Interest: The authors declare that the research was conducted in the absence of any commercial or financial relationships that could be construed as a potential conflict of interest.

Publisher's Note: All claims expressed in this article are solely those of the authors and do not necessarily represent those of their affiliated organizations, or those of the publisher, the editors and the reviewers. Any product that may be evaluated in this article, or claim that may be made by its manufacturer, is not guaranteed or endorsed by the publisher.

Copyright (c) 2021 Zuo, Wang, Chen, Lu and Bai. This is an open-access article distributed under the terms of the Creative Commons Attribution License (CC BY). The use, distribution or reproduction in other forums is permitted, provided the original author(s) and the copyright owner(s) are credited and that the original publication in this journal is cited, in accordance with accepted academic practice. No use, distribution or reproduction is permitted which does not comply with these terms. 